\title{
3D Thermal Imaging: An approach towards true field temperature measurement
}

\author{
by Samuel Petrocelli $1^{1}$, Thierry Sentenac ${ }^{2}$, Yannick Le Maoult ${ }^{3}$ \\ 1,2,3 Ecole des Mines d'Albi, ICA, 81000 Albi, France, sentenac@mines-albi.fr, lemaoult@mines-albi.fr \\ 1,2 Laboratory for Analysis and Architecture of Systems, 31400 Toulouse, France, spetroce@laas.fr
}

\begin{abstract}
There are still many assumptions in the calculation of temperature using infrared cameras, some of which who are dependent on the shape of the observed object and its orientation with the camera. In this paper we address temperature variation occurring as a result of emissivity variance caused by change in the observation angle of an observed object. The camera-object orientation is retrieved by means of 3D reconstruction via a stereo NIR camera rig, which doubles as a redundant thermography system. Examples are given that show the effect of temperature variation with the observation angle variations. We then propose a method for using a modified emissivity measurement method to correct temperature measurement errors dependent on the observation angle.
\end{abstract}

\section{Introduction}

Infrared thermography is becoming a widely available and used technique for temperature measurement. An infrared camera operates in a similar way to most consumer digital cameras, but with the exception that it operates in the infrared light band. Since all objects above absolute zero emit infrared energy, which is correlated with the temperature of the object through well known radiation laws, one can measure the objects temperature using an infrared camera. This is done by converting the infrared image to a temperature map using a calibration law. That is to say, a thermal camera is a thermally calibrated infrared camera.

A common error in this temperature measurement arises from the fact that not all objects being measured have the same infrared emittance for a particular temperature. How an object emits energy is characterized by properties of the objects surface known as emissivity. Temperature measurement with commercial thermal cameras typically requires the user to input or select a constant emissivity value for the particular material type being measured. This greatly improves temperature measurement accuracy, but neglects the fact that emissivity itself can vary depending on the angle at which a particular surface element is being viewed. This paper addresses temperature measurement error in infrared thermography caused by the variance of emissivity in traditional calibration laws that assume emissivity is constant with the observation angle. Given the radiance temperature measurement of the surface element and the observation angle of this element, we can compute a more true temperature. Two thermally and stereo calibrated NIR cameras are used to obtain both the radiance temperature and observation angle at each stereo $3 \mathrm{D}$ point.

The problem of correcting temperature measurement based on the variance of emissivity with the viewing angle is not new either. Various methods are tested for correction of planetary surface temperatures based on the variation of the emissivity dependent on the viewing angle in [4] and [5]. In [6], Sobrino and Cuenca utilize dual angle imagery from the ERS-1 satellite with a dual-angle algorithm to compute emissivity variance on several different surface types (eg. clay, sand). In [7], laniro and Cardone use two infrared cameras in a stereo arrangement where each has a unique wavelength via a narrow band filter. The images from the two infrared cameras are then overlayed on an object of known shape at a steady state temperature. This provides a directionality for each digital value. A system of equations is then solved where emissivity and temperature are the unknown quantities. The result provides the temperature of the object and the directional emissivity of the materials surface. This system handles temperature correction through the estimation of directional emissivity well, but neglects to utilize the stereo infrared cameras as a means of 3D reconstruction.

We approach angular emissivity correction by first measuring the observed objects emissivities using specialized hardware and a spectrometer. These measurements can then be directly applied to the radiance temperature measurement. Later we introduce a method which adapts the thermoreflectometry method for use with stereo infrared cameras. This allows for dual radiance temperature temperature and emissivity measurements.

In addition, using thermal imaging in conjunction with 3D vision is also not a new problem. In [1], Prakash et. al. performed high resolution stereo vision with trichromatic cameras that were calibrated with a single infrared camera where the computed thermal image was overlayed on the point cloud computed by the trichromatic cameras producing a 3D thermal point cloud. They also performed stereo vision directly with two infrared cameras using isotherms as correlation features to improve the thermal point cloud resolution. In another approach, a visible spectrum camera and thermal camera were aligned on the same optical axis, using a germanium window, coupled with a Youngs double split laser (632.8 $\mathrm{nm}$ ) interference pattern [2]. It was even showed possible to use a single camera to reproduce a 3D thermogram, in [3], with a process known as shape-from-heating. The encoded interference pattern is read by the visible camera and used to generate a depth map which the thermal profile is laid upon. In these works, the concept is essentially to perform a stereo $3 \mathrm{D}$ reconstruction of an object via infrared camera(s). That is, shape information is not used to correct temperature errors, rather, the goal is to simply provide a thermal point cloud. 
We propose the use of two stereo calibrated NIR cameras to produce a point cloud of the observed object. The orientation of the surface normal for each point can then be computed based on its spacial relationship to its neighboring points. Given the extrinsic camera parameters and the point normal's, the orientation of each object point can be calculated with respect to the camera. For 3D reconstruction, it is necessary to correlate unique and distinct image features, but with low resolution (commonly 256 x 320) infrared cameras (compared to modern consumer visible digital cameras), these types of features are not prevalent. The use of a projected infrared random pattern on the object and a robust feature detection system aid in the stereo correlation process. Additionally, due to severe image sensor noise, several techniques are described that handle the noise removal from the infrared cameras raw images. Figure 1 depicts the fundamental system which will be detailed by this work.

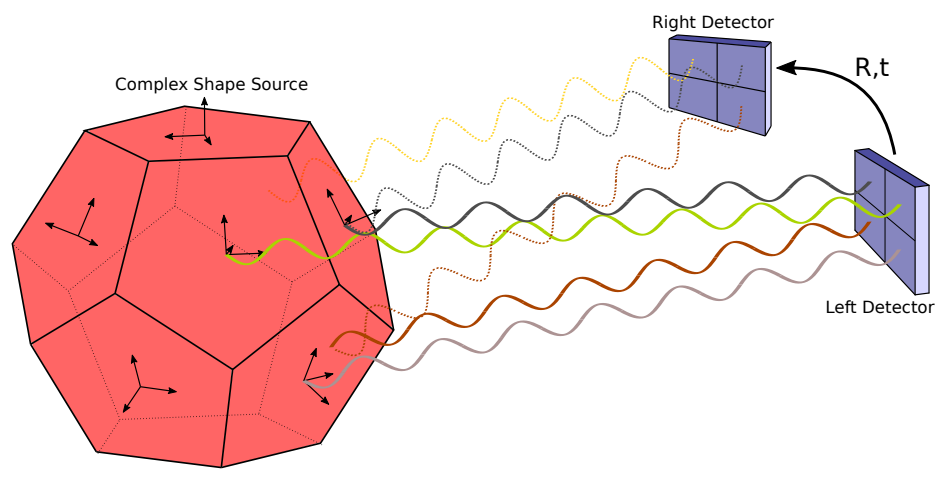

Figure 1: Illustration of proposed system. Two detectors with known extrinsic parameters measuring radiance temperatures of a complex and convex object.

To begin we discuss the true temperature equation and how angular dependent emissivity incorporates into this equation. Next, we give an overview of the thermal calibration law used in our experiemtns and a comparison of it with some other methods. We then move onto the vision segment of this paper, which describes the techniques necessary for performing stereo reconstruction with stereo NIR cameras. From there we introduce a series of experriments that depict the effects of temperature measurement while varying the observation angle of a single NIR camera with planar heated surfaces. Finally, prospects of adapting a method utilizing infrared laser light and camera filters for measuring emissivity is given.

\section{True Temperature Measurement}

The radiance temperature $T_{R}$ is the true temperature for a blackbody and therefore is not the true temperature for all non blackbody objects. Here, we introduce the formula for true temperature measurement. First, we establish the relationship of blackbody radiance $L_{b}(\lambda, T)$, blackbody radiance temperature $T_{R}$, emissivity $\varepsilon$, and true surface temperature $T$

$$
L_{b}\left(\lambda, T_{R}\right)=\varepsilon(\lambda) L_{b},(\lambda, T)
$$

Equation 1 states that an object at temperature $T$ and wavelength $\lambda$ has a blackbody radiance at temperature $T_{R}$ that is equal to the product of the blackbody radiance at it's true temperature and it's emissivity. This is true because for the same temperature and wavelength a non blackbody will radiate less than a blackbody. Typically the radiance $L$ is defined through Planck's Law, but can be substituted for Wien's Approximation for a percent difference of $1 \%$ from Plancks Law when $\lambda \cdot T$ is less then $3100 \mu \mathrm{m} \cdot \mathrm{K}$. For our NIR band of $900 \mathrm{~nm}$ to $1700 \mathrm{~nm}$ and temperature range of $550 \mathrm{~K}$ to $1250 \mathrm{~K}$, this is approximately 467 to $2125 \mu m * K$ with a maximum radiance error of $0.115 \%$. Wien's law for blackbody radiance $L_{b}$ is defined as

$$
L_{b}(\lambda, T)=\frac{C_{1}}{\lambda^{5}} \frac{1}{\exp \left(\frac{C_{2}}{\lambda T}\right)}
$$

where $C_{1}=2 h c^{2}$ and $C_{2}=h c / k . c$ is the speed of light, $h$ is Planck's constant, and $k$ is Boltzmanns constant.

Given equations 1 and 2, we arrive at an invertible equation that yields true temperature as a function of the radiance temperature and emissivity.

$$
\frac{1}{T}=\frac{1}{T_{R}}+\frac{\lambda}{c_{2}} \times \ln (\varepsilon(\lambda))
$$

A true temperature measurement is, therefore, dependent on a radiance temperature and thermal properties of the materials surface. 
With the equation for true temperature based on the objects emissivity and radiance temperature defined, we can now observe how these quantities behave, in sections 2.1 and 2.2, in relation to the observation angle. That is, we will redefine the emissivity and radiance temperature components to be $\varepsilon^{\theta}(\lambda)$ and $T_{R}($ theta $)$.

\subsection{Emissivity versus Observation Angle}

In our research we explore using specialized apparatus for indirectly measuring the hemispherical and directional emissivity of a particular material. Assuming the sample is in thermal equilibrium and is opaque, Kirchoff's Law allows the computation of the emissivity from the reflectivity

$$
\varepsilon\left(\lambda, \theta_{i}\right)=1-\rho^{\theta_{i}, \cap}(\lambda, T)
$$

A spectrometer fitted with a variable angle reflectivity measurement accessory, similar to a gonioreflectometer allows setting a distinct incident angle that a beam of infrared light will approach a sample and then measures distinct reflectivity points at varying angles. For each incident angle, many reflectivity measurements are taken along a series of angles. These reflectivity measurements are integrated to produce a hemispherical reflectance that is specific to an incident angle.

$$
\rho^{\theta_{i}, \cap}(\lambda, T)=\int_{\Omega=2 \pi} \rho^{\theta_{i}, \theta_{r}} \cos \left(\theta_{r}\right) d \Omega
$$

where $i$ is the incident angle. According to equation 4 and the integrated reflectance measurement, we can now compute the emissivity $\epsilon\left(\lambda, \theta_{i}\right)$. That is, for each incident angle we have computed the emissivity at that angle. Since the geometry of the mirrors in the measuring device do not obtain measurements for a distinct point, we must use an emissivity value computed from a spherical reflectance to interpolate the directional emissivities. That is, the spherically obtained emissivity acts as a reference emissivity for what $\varepsilon(\theta=0)$ should be. The final result is the integration scaled by a interpolation factor $K$ and in combination with equation 4

$$
\varepsilon\left(\lambda, \theta_{i}\right)=1-K \int_{\Omega=2 \pi} \rho^{\theta_{i}, \theta_{r}} \cos \left(\theta_{r}\right) d \Omega
$$

The resulting $\epsilon\left(\lambda, \theta_{i}\right)$ values can be used by equation 8 for true temperature computation given the observation angle and radiance temperature at the appropriate wavelength.

\subsection{Radiance Temperature versus Observation Angle}

Traditionally, the radiance temperature has a projective dependency on the viewing angle as seen in Figure 2 . The projective radiation intensity $I$ varies with the cosine of the surface radiation intensity $I_{0}$.

$$
\frac{I_{D}}{I_{D_{0}}}=\cos (\theta)
$$

This holds when the surface area element captured by a pixel is always the same. Though, as is, the conical projection of a single image sensor (pixel) onto an observed objects surface will produce an elliptical surface area that increases with the observation angle. To obtain the same radiance temperature, irregardless of observation angle, the projected surface area needs to be normalized with respect to the projected zero normal angle $(\theta=0)$ area $A^{0}$. First, we define the projected area $A$ as

$$
A=\pi a b
$$

where $a$ and $b$ are the semi-major axis of the projected ellipse. To obtain $A^{0}$ we say

$$
a=b=\ell \tan (\alpha)
$$

where $\alpha$ is the field-of-view of a single image sensor detector and $\ell$ is the distance extending through the projected conic center from the detector to the objects surface. When $\theta$ is greater than zero

$$
a=\ell \sin (\alpha)\left(\frac{1}{\sin \left(\gamma_{1}\right)}+\frac{1}{\sin \left(\gamma_{2}\right)}\right) \quad \text { and } \quad b=\ell \tan (\alpha)
$$

where $\gamma_{1}=90-\theta-\alpha, \gamma_{2}=90+\theta-\alpha$, with the assumption that $\theta<90-\alpha$.

Given an isotropic and uniform surface, the incident flux on the detector would increase by a factor of $A / A^{0}$ as the observation angle increases. This increase in flux on the detector would result in a higher pixel digital level and ultimately a higher radiance temperature. The solution is to normalize the detectors digital level by a factor of $A^{0} / A$. This theory will be tested in section 6 by a commercially available flat black body.

In conclusion, we can now redefine the model for true temperature in a way that it is dependent on the observation angle outlined in sections 2.1 and 2.2. This equation combined with the radiance temperature measurement and observation angle retrieval methods outlined in the sections to follow allow for a simple and robust approach towards true temperature measurement.

$$
\frac{1}{T}=\frac{1}{T_{R}(\theta)}+\frac{\lambda}{c_{2}}+\ln (\varepsilon(\lambda, \theta))
$$




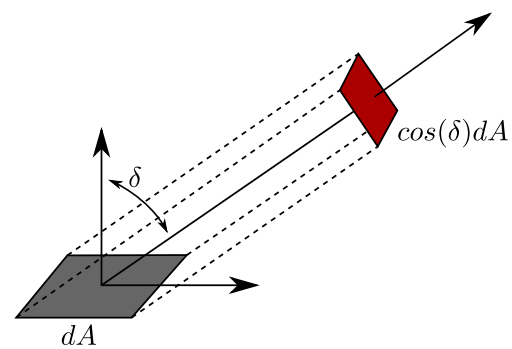

Figure 2: Surface area element $d A$ projection.

\section{Radiometric Calibration of NIR Cameras}

Thermal calibration begins by acquiring images of a commercially available blackbody at different temperatures with the infrared camera. A model is then used to correlate each images mean pixel value with the images respective blackbody temperature. The extended wavelength model found in [8] was used as the calibration model and is well adapted to our temperature and spectral range

$$
I_{D_{0}}(u, v)=\frac{D}{\exp \left(\frac{C_{2}}{\lambda_{x}\left(T_{R}, u, v\right) \times T_{R}}\right)}
$$

with

$$
\frac{1}{\lambda_{x}\left(T_{R}, u, v\right)}=a_{0}(u, v)+\frac{a_{1}(u, v)}{T_{R}}\left(+\frac{a_{2}(u, v)}{T_{R}^{2}}\right)
$$

where $I_{D}$ is the temporally averaged image sensor value, $D$ is the camera specific spectral response fitting parameter, $C_{2}$ the radiation constant equal to $14388 \mathrm{~K} \mu \mathrm{m}, \lambda_{x}$ is the extended effective wavelength, and $T_{R}$ is the radiance temperature in degrees Kelvin. To continue, this model can be linearized through log expansion to simplify it into a polynomial expression

$$
\log \left(\frac{I_{D_{0}}}{t_{i}}\right)=\log (D)-c_{2} a_{0} \frac{1}{T_{R}}-c_{2} a_{1} \frac{1}{T_{R}^{2}}\left(-c_{2} a_{2} \frac{1}{T_{R}^{3}}\right)
$$

where $t_{i}$ is the integration time. We assume that the response of the analog to digital converter (ADC) of each detector of the infrared camera behaves linearly at a digital pixel value of 75 percent of the total pixel range in respect to variation of the integration time. With this assumption, we can normalize $\bar{I}_{D}$ with respect to the integration time as shown in Eq. 11 . This allows us to work within a much greater temperature range and not be bound by a single integration time. Furthermore, this linearly deduced form can be easily solved for the radiance temperature $T_{R}$ using a linear system of equations solver such as SVD. The radiance temperature is the measured temperature for a blackbody.

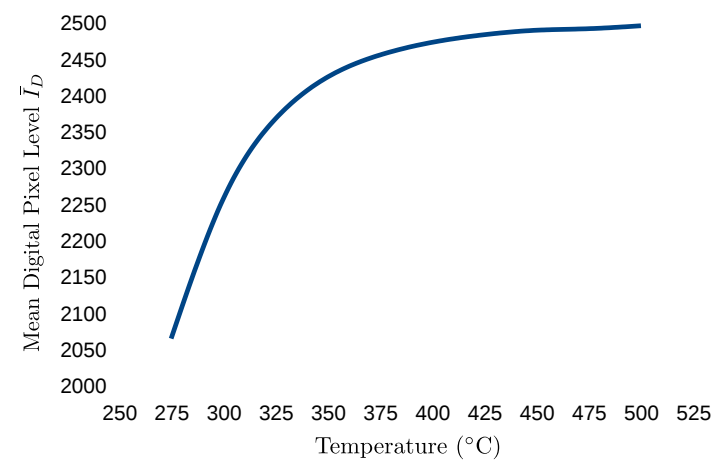

Figure 3: Thermal calibration result using the extended wavelength model.

\subsection{Radiometric Calibration with Observation Angle}

To incorporate the effects of apparent surface area as seen in Figure 2, it is necessary to modify equation 11 such that it uses $I_{D}$ as it's digital intensity

$$
\log \left(\frac{\bar{I}_{D}}{t_{i}}\right)=\log (D)-c_{2} a_{0} \frac{1}{T_{R}}-c_{2} a_{1} \frac{1}{T_{R}^{2}}\left(-c_{2} a_{2} \frac{1}{T_{R}^{3}}\right)
$$


where $I_{D}=I_{D_{0}} \cos (\theta)$. This means that each pixel of the camera must view the same surface area of the thermal calibration target. A simplification of this can be performed by taking a mean pixel value of a specific area on a flat calibration target. This would involve marking an area on the target that is detected by software and the mean pixel value taken of that physically specfied area. As the target is rotated, the projected area as seen by the camera will reduce with the cosine of the observation angle. This is the theoretically appropriate way to perform a radiometric calibration that handles observation angle. Do to time and limitations of hardware, the method used by this paper is one which performs radiometric calibration on with $I_{D_{0}}$.

\subsection{Survey of Non-Physical Radiometric Calibration Models}

The radiometric calibration model described by equation 11 is derived from physical radiometric laws, and in this section we compare its performance with more conventional regression methods. Performance testing involves validating the models ability to fit radiance temperature verse digital pixel intensities. Polynomial and power function based models were also tested where the functions accepted both $I_{D}$ and $t_{i}$ as parameters. For polynomials the following model was tested

$$
T_{R}\left(I_{D}, t_{i}\right)=\sum_{j=0}^{n} a_{j} I_{D}^{j}+\sum_{k=0}^{m} b_{k} t_{i}^{k}
$$

where $\mathrm{n}$ and $\mathrm{m}$ are the degrees of the sub-polynomials governing the independent regression of both $I_{D}$ and $t_{i}$, such that $n \geq 0$ and $m \geq 0$, and $a_{j}$ and $b_{k}$ are the respective coefficients of the sub-polynomials. This method could not became under determined before a large enough polynomial degree could be achieved to match the data profile. The second method of power function fitting showed good results. After experimentally determining the natural trend of the 3D plot of $\left(T_{B B}, I_{D}, t_{i}\right)$ it was seen that the following model fit the data quite well.

$$
T_{R}\left(I_{D}, t_{i}\right)=a I_{D}^{b} t_{i}^{c}
$$

where $a, b$, and $c$ are the estimated coefficients. Table 1 shows the results for performing the thermal calibration using each of the proposed methods. In order, there is the polynomial model with $m=n=4$, the power function, the degree 2 extended wavelength model, and finally the degree 3 extended wavelength model. The results in Table 1 were performed with a mean pixel value of $75 \%$ where, as mentioned, the camera behaves linearly with the integration time.

\begin{tabular}{|l|c|c|c|c|}
\hline Func & Min & Max & Mean & Std. Dev. \\
\hline \hline Poly & 0.000 & 5.025 & 1.447 & 1.805 \\
Power & 0.043 & 0.733 & 0.312 & 0.185 \\
E.W. 2 & 0.003 & 1.074 & 0.446 & 0.266 \\
E.W. 3 & 0.004 & 0.101 & 0.038 & 0.034 \\
\hline
\end{tabular}

Table 1: Thermal calibration results for a single digital pixel level.

It is clear that the degree 3 extended wavelength model produces the most accurate radiance temperature measurement. However, Table 2 shows the results for same set of Functions, but using a series of temperature measurements over several mean pixel value percentages (25 to 75 percent with 10 percent intervals). The power function was specifically chosen to handle the non-linearity of the integration time vs digital pixel level and therefore produces the most accurate radiance measurement out of the three methods.

\begin{tabular}{|l|c|c|c|c|}
\hline Func & Min & Max & Mean & Std. Dev. \\
\hline \hline Poly & 0.377 & 73.058 & 20.293 & 13.29 \\
Power & 0.001 & 3.052 & 1.191 & 0.755 \\
E.W. 2 & 0.063 & 14.717 & 4.63 & 3.323 \\
E.W. 3 & 0.040 & 14.244 & 4.603 & 3.319 \\
\hline
\end{tabular}

Table 2: Thermal calibration results for a series of digital pixel levels.

\section{Infrared Stereo Vision}

To determine the observation angle of each surface element of the object, we use a stereo infrared rig which provides a 3D reconstruction of the observed object. Since the camera poses are known with respect to the 3D reconstruction, we can recover the observation angle of the camera for each 3D point. That is, for each 3D point $(x, y, z)$ there are two digital 
intensities, one from each of the cameras, and an observation angle $\theta$. This observation angle is used in conjunction with it's two respective digital intensities to calculate a temperature at $(x, y, z)$.

To begin, the pin-hole camera model is used to approximate of the behavior of the infrared cameras lens and detector system. With the two cameras arranged in a typical stereo configuration, stereo calibration is performed using a checkerboard pattern that is illuminated with a tungsten lamp. This step first computes image transformations necessary to remove tangential, spherical, and prismatic lens distortions and then computes the translation and rotational matrices between the cameras. To follow, we need to perform stereo correspondence which is one of the main problems in stereo vision. Here we match unique features in the left and right image, but given the low resolution of the cameras and lack of features [9], standard techniques used in tri-chromatic stereo correlation do not work well. In [10], it is shown that random SIFT [11] image features can be matched between the two images to perform a sparse, but robust image correlation. Features are matched via a brute force matching assisted by a cross referenced point to epipolar line distance constraint. In addition to robust feature extraction and matching, an IR LED operating at $940 \mathrm{~nm}$ is projected though a random cloud mask onto the observed object. This greatly improves the likelihood of uniquely observable features on the observed object.

To extract the observation angle it is necessary to determine the pose of each 3D point. For this, the PCA (principle component analysis) of $n$ nearest neighbors for each point is calculated. This produces a 3 vector orthonormal basis, known as the eigenvectors of the system, and the magnitudes of these vectors as eigenvalues. The eigenvector associated with the smallest eigenvalue is a statistical approximation of a points normal. Given the normal and camera geometry, the observation angle can be computed.

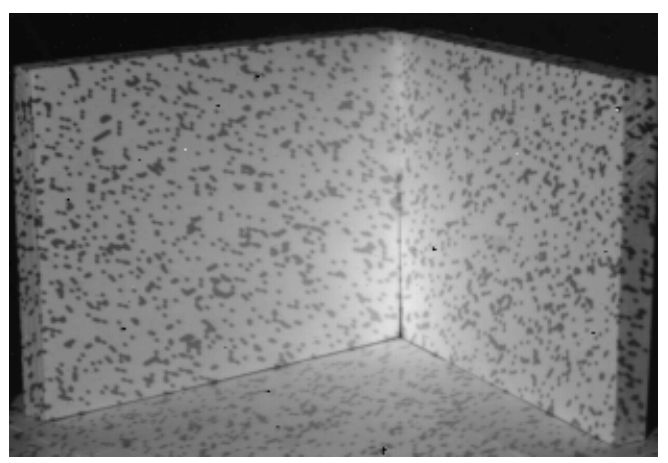

Figure 4: Random cloud projected onto two white planes. The black speckle is the projected IR light shadowed by the mask.

\subsection{Image Noise Removal}

The raw frames acquired from the cameras contain both dead pixels and vertical active striping (detector noise floor) whose intensity positively covaries with the integration time. Dead pixels are removed by acquiring many images of scenes of varying intensities and calculating the variance for each pixel location across this series of images. Clustering shows two distinct groups of high and low variance pixels. Low variance pixels are restored using a generic proximity image restoration algorithm. Active striping is removed by acquiring a series of dark images with increasing integration time. For each pixel location, a polynomial is computed that represents the noise images pixel value the integration time the image was taken at. Now, given a raw camera frame and its integration time, the noise image can be computed and subtracted from the raw image.

\section{System}

To summarize, for each matched point from the two NIR cameras we have the data vector $\left[x, y, z, I_{D 1}, S_{D 2}\right]$, where $(x, y, z)$ is the 3D point calculated from the stereo reconstruction and $\left(S_{D 1}, S_{D 2}\right)$ are the digital pixel values from the left and right cameras, respectively. From this, we can infer the data vector $\left[T_{R 1}, T_{R 2}, \theta_{1}, \theta_{2}\right]$; the radiance temperatures of the 3D point and the orientation of the 3D points normal with the left and right cameras, respectively. This is then used to compute a true temperature at the particular 3D point using the equation for true temperature discussed in section 2.

\section{Experimental Illustration of View Angle Influence on Temperature Measurement}

In this section simple objects radiant temperature is measured in relation to the angular orientation between the objects normal and the cameras optical axis. Angles are measured from the optical axis in degrees. 
As a a baseline test, the temperature variance of a commercially available blackbody heated to $360^{\circ} \mathrm{C}$ is measured in relation to the observation angle. The test is performed using a Xenics NIR camera calibrated using the extended wavelength model described earlier. The temperature is measured by taking the mean temperature over a selection of pixels on the surface of the blackbody. Figure 5 shows the results of this experiment. There are two components being tested by this experiment. Is the blackbody isotropic (constant emissivity of 1 ) and does the projected pixel area described in 2.2 show a significant effect.

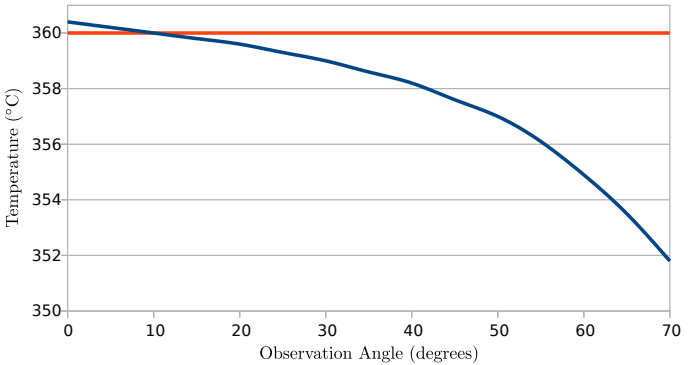

Figure 5: Commericial flat blackbody temperature with the change in observation angle.

As expected, it is seen that the commercial blackbody behaves realistically isotropic. To note, the difficulties in measuring temperatures at shallow observation angles occurs as a result of trying to average a temperature over a small vertical selection of pixels in the temperature image. In this experiment and the experiments to follow it is rather inconclusive if the radiance temperature measurements at angles beyond 75 degrees from the normal are completely accurate. Given the consistent decrease in temperature with the angle, it can also be concluded that projected pixel area does not play a significant role in temperature computation. The remaining duration of this paper will assume that the radiance temperature $T_{R}$ does not effected by observation angle.

The next experiment is performed in a similar fashion, but using a solid copper plate with an even and slightly roughened silver paint coating. Figure 8 shows the apparatus used for measuring the temperatures. Here both cameras are shown, but the results provided for this experiment only utilize one camera. Here we are testing to see if the emissivity of the silver paint behaves like a classical metallic surface where the emissivity increases with the angle from the normal (See Figure $6)$.

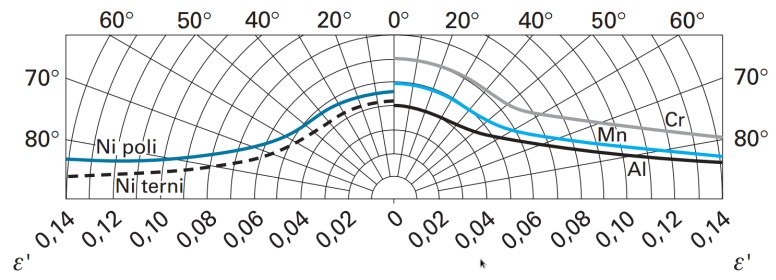

Figure 6: Variation of emissivity at several wavelengths with the observation angle for some materials [12].

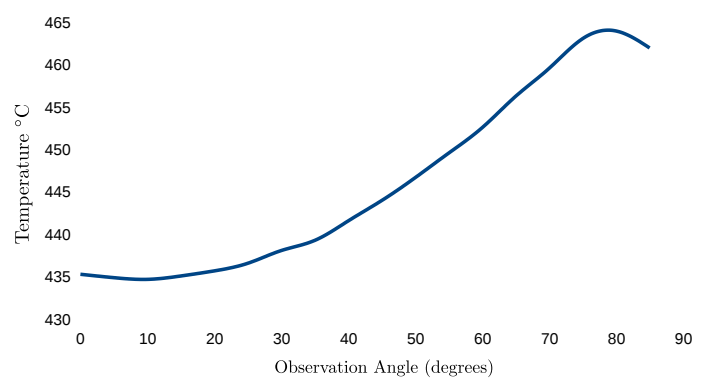

Figure 7: Silver painted copper plate temperature with the change in observation angle.

The measured temperature increases with the observation angle, which coincides with the increase of emissivity with the observation angle. It was important to perform this experiment in a timely fashion as to not allow the paint to oxidize. If oxidation were to occur, the paint would not have been able to perform as a metallic surface. 


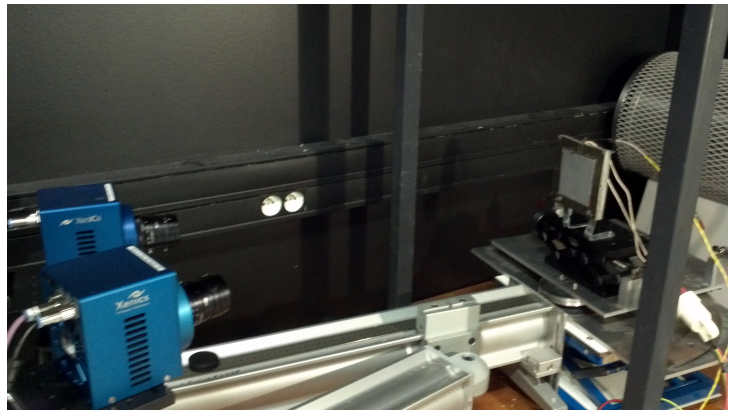

Figure 8: Silver painted copper plate temperature with the change in observation angle.

\subsection{Incorporating Emissivity}

Our first approach in evaluating and incorporating emissivity is to indirectly measure it using a spectrometer fitted with a variable angle reflectance measurement accessory. The results of this technique can be seen in graph 9 , which shows reflectance measurements at different angles and for several different wavelengths. This method is currently being evaluated as to it's effectiveness in accurately accounting for angular emissivity measurement.

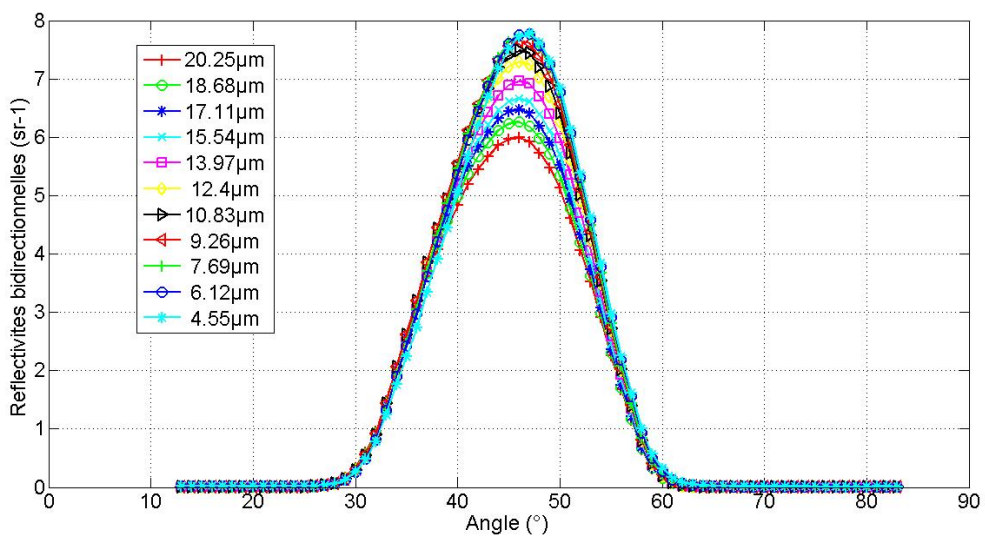

Figure 9: Reflectance measurements from the spectrometer

\subsubsection{Measuring Emissivity via Infrared Cameras}

The emissivity can be determined through a model approach [7] or measured directly [13]. Modeling would require providing a universally acceptable model for the variance of emissivity with the viewing angle. [7] achieved modeling emissivity using a model proposed by [14] which is derived from Fresnel equations in conjunction with Kirchoffs laws. These equation assume the surface is opaque and is isotropic with no dependence on the azimuthal angle. It is thought of a poor approximation due to the assumption that the material is isotropic.

Measuring emissivity, as shown in [13], which is based on the work of [15], can be achieved by a method known as bicolor near infrared thermoreflectometry which measures the true temperature of a flat opaque surface of unknown emissivity. A single static NIR camera operating at two independent wavelengths measures the radiance temperature of the object with and without two laser sources operating at wavelengths respective to the two filters applied to the camera. Since the reflectance of a material is related to the emissivity (Eq. 15), measurement of the reflectance at two different wavelengths allows for an indirect measurement of the emissivity. They also show that neglecting to use the correct emissivity at a high viewing angle in pyrometry can lead to a temperature measurement error of $30^{\circ}$.

$$
\varepsilon^{\vec{r}_{0}}(\lambda, T)=1-\rho^{\vec{r}_{0}, \cap}(\lambda, T)
$$

It would be ideal that our system modify the method in [13] to use two cameras operating each at a separate wavelength. The thermoreflectometry comes with the assumption that the object being viewed is flat and has a static orientation with the camera such that the reflected laser light has the same geometry for both lasers. Adapting thermoreflectometry with our two camera system, such that each camera operates at a respective wavelength, the reflection of the laser light would then also depend on the geometry of the system. It is likely that this is not possible as it would require characterizing the reflectivity of the material with the observation angle before performing the experiment. The more likely case is applying a filter wheel to each camera that allows both to operate at the two wavelengths. This method would provide dual NIR 
thermoreflectometry measurements and allow direct cross referencing and error analysis of each measurement for the same 3D point.

\section{Conclusion}

In this paper it was shown how the emissivity depends on the angle of observation through experimental results. An overview of the infrared stereo vision was given and the methods to overcome sparse detection of dull features. With the extended wavelength model to provide the radiant temperature, true temperature measurement was shown possible using a modified thermoreflectometry system. The continuation of this work will be evaluating the proposed dual thermoreflectometry method.

\section{References}

[1] Surya Prakash, Pei Yean Lee, and Antonio Robles-Kelly. Stereo techniques for 3d mapping of object surface temperatures. QIRT, 4(1):63-84, January 2007.

[2] Hai Xiao, Yibing Zhang, and Anbo Wang. Multispectral three-dimensional digital infrared thermal imaging. Society of Photo-Optical Instrumentation Engineers, 42(4):906-911, April 2003.

[3] Jean-Francois Pelletier and Xavier Maldague. Shape from heating: a two-dimensional approach for shape extraction in infrared images. Society of Photo-Optical Instrumentation Engineers, 36(2):370-375, February 1997.

[4] Zhao-Liang Li, Hua Wu, Ning Wang, Shi Qiu, Jos A. Sobrino, Zhengming Wan, Bo-Hui Tang, and Guangjian Yan. Land surface emissivity retrieval from satellite data. International Journal of Remote Sensing, 34(9-10):3084-3127, August 2013.

[5] Jose A. Sobrino and Juan Cuenca. Angular variation of thermal infrared emissivity for some natural surfaces from experimental measurements. Optical Society of America, 38(18):3931-3936, June 1999.

[6] Juan Cuenca and Jose A. Sobrino. Experimental measurements for studying angular and spectral variation of thermal infrared emissivity. Optical Society of America, 43(23):4598-4602, August 2004.

[7] A. laniro and G. Cardone. Measurement of surface temperature and emissivity with stereo dual-wavelength ir thermography. Journal of Moderm Optics, 57(18):1708-1715, October 2010.

[8] Thierry Sentenac and Remi Gilblas. Noise effect on the interpolation equation for near infrared thermography. Metrologia, 50:208-218, May 2013.

[9] Kiana Hajebi and John Zelek. Structure from infrared stereo images. In Fifth Canadian Conference on Computer and Robot Vision, Windsor, Ontario, May 2008.

[10] Benot Ducarouge. These: Reconstruction 3d infrarouge par perception active.

[11] David G. Lowe. Object recognition from local scale-invariant features. In Seventh International Conference on Computer Vision.

[12] Simone Mattei. Rayonnement thermique des matriaux opaques. Technical report, l'Universit de Bourgogne.

[13] Thierry Sentenac, Rmi Gilblas, Daniel Hernandez, and Yannick Le Maoult. Bi-color near infrared thermoreflectometry: A method for true temperature field measurement. Review of Scientific Instruments, December 2012.

[14] Robert Siegel and John R. Howell. Thermal Radiation and Heat Transfer. Hemisphere Publishing Corporation, 3rd edition, 1992.

[15] D. Hernandez, J.M. Badie, F. Escourbiac, and R. Reichle. Development of two-colour pyroreflectometry technique for temperature monitoring of tungsten plasma facing components. Fusion Engineering and Design, 2008. 